\title{
Novel Technique for Tar mat identification and mapping
}

\author{
Naim Al-Jabari, Halliburton
}

Tar mat identification and mapping has been a challenge for the oil industry for years. Limited number of techniques and tools are available to identify tar presence in an oil field. These can be divided to three main categories:

- Laboratory based on drilling cuts, core or side cores in suspected tar zone.

- Log based, using mainly NMR and Formation Tester

- Surface and borehole Seismic.

Each of these three main techniques has its own advantages and disadvantages. The first two techniques suffer from limited availability across the field to allow mapping of tar. Furthermore, NMR logs also have difficulty distinguishing tar from heavy oil as both show similar NMR characteristics. Surface and borehole seismic on the other hand are excellent tools for mapping but lacks the ability to accurately identify tar mats.

The Novel technique proposed in this paper combines cheomstratigraphy, NMR, formations tester, geochemical logging and borehole seismic to identify and map tar mats. The main concept behind the new technique is to link tar identification to three independent measurements chemical, NMR and acoustic to overcome limitation associated with each technique. Then use borehole seismic to map tar mats based on the tar attributes defined by laboratory and wireline acoustic measurement. The technique is implemented in three main stages first identifying tar and separating it from any heavy oil that might be associated with it. Second stage, is finding the tar cheomstratigraphic finger print that can be linked to a geochemical log. This stage will allows identification of tar map thickness in well by well bases. The third and final stage, is linking tar acoustic properties measured in both laboratory and wireline to borehole seismic attributes then surface seismic to map tar across the field.

In the first stage, heavy oil and tar are identified using NMR and separated based on mobility. To identify heavy oil/tar, NMR porosity is compared with Density-Neutron porosity. If an under estimation of porosity is observed (deficit porosity), this would be an indication of presence of heavy oil/tar or gas. Gas presence indicator such as neutron can be used to confirm the hydrocarbon type. If heavy oil/tar presence is confirmed, formation tester using slow pump rate can be used to try and collect oil sample. If no movable oil is observed, stimulation gun is used to try to reduce oil viscosity and increase mobility. It is assumed that any heavy oil part that does not show mobility even after stimulation is most likely to be tar. Laboratory analysis will be required to confirm that by taking a side core in the suspected tar zones. It is worth mentioning that the first stage can be implemented by either LWD or WL since NMR and sampling while drilling have been introduced to the industry.

In the second stage, once Tar has been identified. The cheomstratigraphy made on drilling cuttings using LaserStrat is linked to tar zones. The technique assumes that during tar generation a distinct cheomstratigraphic finger print is created. Elements ratios associated with tar obtained from LaserStrat are confirmed by laboratory elemental analysis made on the collected tar sample. The chemical element ratio finger present of tar is then applied to geochemical logs to quantify the thickness of the tar in a particular well.

In the third and final stage, the tar acoustic properties measure in the laboratory and wireline loggings are obtained. The wireline sonic properties calibrated to core data are then correlated to borehole seismic to be able of mapping the tar attributes. This can be further connected to surface seismic to map a tar across the field. 
The proposed technique in this paper does not require the full suite of measurements to be made in all wells. It will require number of key wells representative of reservoir structure. The accuracy of the tar mapping prediction can be monitor using Chemostratigraphy on drilling cuttings and/or geochemical logging in other wells across the field. This will also allow fine tuning of tar mapping across the field. 